ARTICLE

DOI: $10.1038 / \mathrm{s} 41467-018-07288-6$

\title{
Accelerating water dissociation kinetics by isolating cobalt atoms into ruthenium lattice
}

Junjie Mao,2, Chun-Ting $\mathrm{He}^{3}$, Jiajing $\mathrm{Pei}^{4}$, Wenxing Chen², Dongsheng $\mathrm{He}^{5}$, Yiqing $\mathrm{He}^{2}$, Zhongbin Zhuang ${ }^{4}$, Chen Chen ${ }^{2}$, Qing Peng ${ }^{2}$, Dingsheng Wang ${ }^{2}{ }^{2} \&$ Yadong $\mathrm{Li}^{2}$

Designing highly active and robust platinum-free catalysts for hydrogen evolution reaction is of vital importance for clean energy applications yet challenging. Here we report highly active and stable cobalt-substituted ruthenium nanosheets for hydrogen evolution, in which cobalt atoms are isolated in ruthenium lattice as revealed by aberration-corrected high-resolution transmission electron microscopy and X-ray absorption fine structure measurement. Impressively, the cobalt-substituted ruthenium nanosheets only need an extremely low overpotential of $13 \mathrm{mV}$ to achieve a current density of $10 \mathrm{~mA} \mathrm{~cm}^{-2}$ in $1 \mathrm{M} \mathrm{KOH}$ media and an ultralow Tafel slope of $29 \mathrm{mV} \mathrm{dec}{ }^{-1}$, which exhibit top-level catalytic activity among all reported platinum-free electrocatalysts. The theoretical calculations reveal that the energy barrier of water dissociation can greatly reduce after single cobalt atom substitution, leading to its superior hydrogen evolution performance. This study provides a new insight into the development of highly efficient platinum-free hydrogen evolution catalysts.

\footnotetext{
${ }^{1}$ Key Laboratory of Functional Molecular Solids, Ministry of Education, Anhui Key Laboratory of Molecule-Based Materials, College of Chemistry and Materials Science, Anhui Normal University, Wuhu 241000, China. ${ }^{2}$ Department of Chemistry, Tsinghua University, Beijing 100084, China. ${ }^{3}$ MOE Key Laboratory of Functional Small Organic Molecule, College of Chemistry and Chemical Engineering, Jiangxi Normal University, Nanchang 330022, China. ${ }^{4}$ State Key Lab of Organic-Inorganic Composites, Beijing University of Chemical Technology, Beijing 100029, China. ${ }^{5}$ Materials Characterization and Preparation Center (MCPC), South University of Science and Technology of China, Shenzhen, Guangdong 518055, China. These authors contributed equally: Junjie Mao, Chun-Ting He, Jiajing Pei. Correspondence and requests for materials should be addressed to

D.W. (email: wangdingsheng@mail.tsinghua.edu.cn)
} 
$\mathrm{T}$ he electrocatalytic hydrogen evolution, as an attractive strategy to the production of hydrogen, has been receiving great attention in clean alternative energy technologies ${ }^{1-5}$. Generally, the hydrogen evolution reaction (HER) in acidic media is much easier than that in alkaline media. This is because the reaction precursor in acidic media is a proton which can easily receive electrons from the cathode. The sluggish kinetics of the HER, particularly in alkaline electrolytes, calls for developing highly efficient catalysts to lower the hydrogen evolution overpotential. To date, $\mathrm{Pt}$ is the most popular HER catalyst due to its high exchange current density $\left(j_{0}\right)$ and small overpotentials $(\eta)$. However, the scarcity, high price and poor durability of Pt greatly restrict its commercial applications. To address these issues, many research groups have employed various approaches to obtaining Pt-free catalysts with superior HER catalytic activity. For instance, constructing unique nanostructures (nanoframes ${ }^{6,7}$, defect-rich nanosheets/nanowires ${ }^{8-11}$, porous/hybrid nanostructures ${ }^{12-19}$, etc.) with abundant active sites; introducing heteroatoms like $\mathrm{N}$, $\mathrm{P}, \mathrm{S}$ into metal elements to form metal-N/P/S catalysts $^{15,20-22}$; preparing single atom catalysts supported on porous carbon nanomaterials $23-25$; loading catalysts on a proper substrate/ support $^{23,24,26-28}$ and so on. Although much work has been done in this field, only a few of the instances of Pt-free catalysts with optimized structure and composition have shown the satisfactory activity in comparison with Pt. On the other hand, the irregular structure and/or complex compositions of the reported catalysts make it difficult to understand the relationship between catalytic sites at the atomic scale and hydrogen evolution performance. Therefore, it is necessary to develop new strategies for designing novel Pt-free electrocatalysts at the atomic level, with much enhanced catalytic activity and durability for HER than commercial $\mathrm{Pt} / \mathrm{C}$ catalyst.

In this study, we prepare Co-substituted $\mathrm{Ru}$ nanosheets (NSs) with atomically dispersed Co into Ru lattice. Remarkably, the asobtained Co-substituted Ru NSs exhibit superior catalytic activity towards HER in $1 \mathrm{M} \mathrm{KOH}$ media, with an ultralow overpotential of $13 \mathrm{mV}$ to achieve a current density of $10 \mathrm{~mA} \mathrm{~cm}^{-2}$ and an ultralow Tafel slope of $29 \mathrm{mV} \mathrm{dec}^{-1}$, and excellent durability over 1000 cycles. To our knowledge, the Co-substituted Ru NSs exhibit the top-level catalytic activity for HER among all reported Pt-free electrocatalysts. The experimental results and density functional theory (DFT) calculations further demonstrated that single Co atom substituted $\mathrm{Ru}$ catalysts can remarkably reduce the energy barrier of water dissociation in comparison with pristine $\mathrm{Ru}$ and RuCo alloy, thus leading to superior performance toward HER.

\section{Results}

Synthesis and characterizations. In a typical synthesis, Cosubstituted Ru NSs were synthesized by co-reduction of $\mathrm{Ru}(\mathrm{acac})_{2}$ and $\mathrm{Co}(\mathrm{acac})_{2}$ in the mixed solutions containing oleylamine and heptanol at $180^{\circ} \mathrm{C}$ for $12 \mathrm{~h}$. Transmission electron microscopy (TEM) and high-angle annular dark-field scanning TEM (HAADF-STEM) were conducted to investigate the morphology and structure of the as-obtained products. As shown in Fig. 1a and Supplementary Fig. 1, the uniform hexagon NSs with a lateral size around $30 \mathrm{~nm}$ were synthesized. Interestingly, the obtained nanosheets can self-assemble into patterned arrays (Supplementary Fig. 2) when depositing high concentration of nanosheets on the copper grid. Atomic force microscopy was utilized to determine the thickness of NSs (Fig. 1b). Section analysis and height profile show that the thickness of the obtained NSs is about $\sim 1.6$ $\mathrm{nm}(\sim 7$ atomic layers of $\mathrm{Ru})$. The atomic structure of nanosheets is further examined by atomic resolution aberration-corrected HAADF-STEM imaging technique. Figure $1 \mathrm{c}$ shows that the obtained NSs have the single-crystalline nanostructure, which possesses the side plane of $\mathrm{Ru}(01 \overline{1} 0)$ facets, and the basal plane of $\mathrm{Ru}$ (0001) facets. Moreover, the enlarged image of the surfaces of nanosheet also reveals that the Co atoms locate at the hexagonal close-packed (hcp) Ru lattice positions. Figure 1d shows the HAADF line scanning profile of Co-substituted Ru NSs. Figure 1e

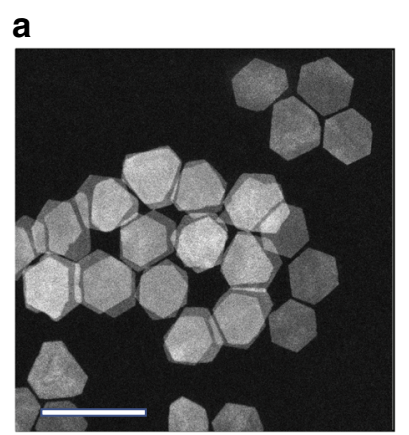

b
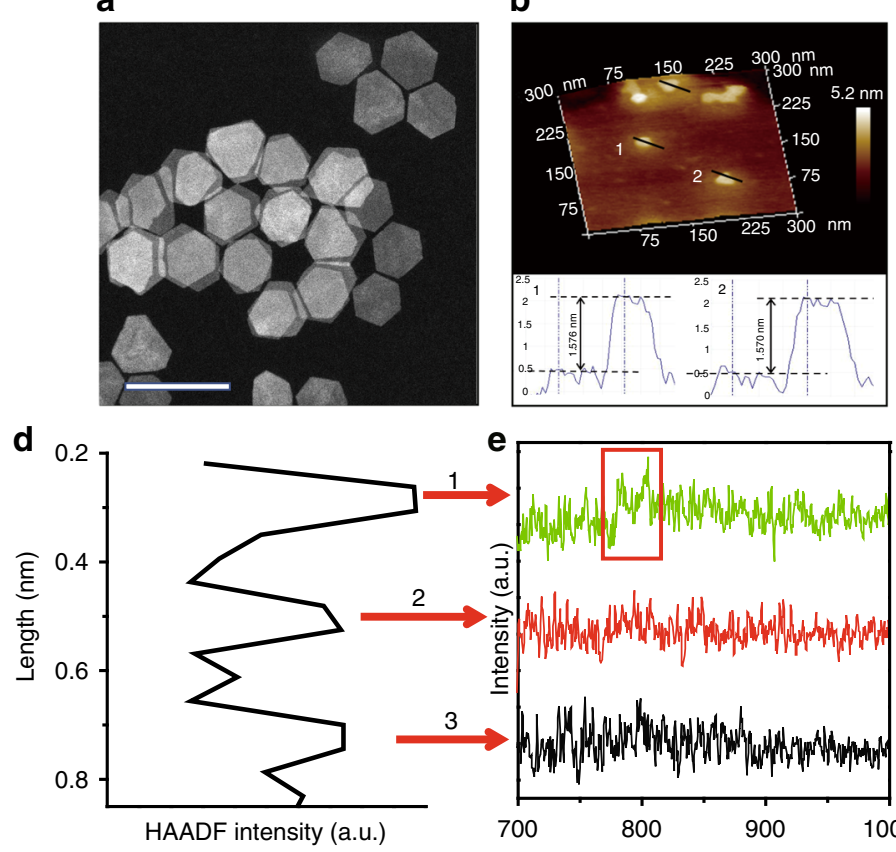
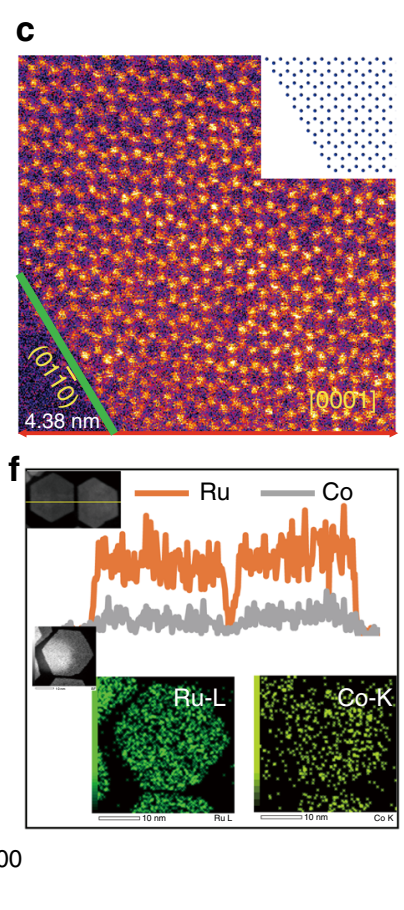

Fig. 1 Characterization of Co-substituted Ru NSs. Representative a STEM image. Scar bar: 50 nm. b AFM image. c Atomic resolution HAADF-STEM image

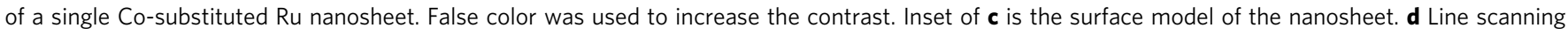
profile and $\mathbf{e}$ background subtracted EELS spectrum of the Co-substituted Ru NSs acquired from $\mathbf{d}$. The red rectangle represents Co $L_{2}$, 3 -edge signal. $\mathbf{f}$ EDX line scanning profile and EDX mapping of a single Co-substituted Ru NS 
shows the simultaneously acquired electron energy loss spectroscopy (EELS) spectra of the Co K-edge taken from the three atomic column locations indicated in Fig. 1d. We only found that position 1 exhibits typical $\mathrm{Co}_{2}$, 3 -edge signal, while we could not observe the similar signals from neighboring atoms, indicating a highly monodispersed distribution of Co atoms in the NSs. The X-ray diffraction (XRD) pattern (Supplementary Fig. 3) of the NSs could be assigned to (10ī0), (0002) and (10īi) diffractions of a hcp structure. No obvious diffraction peak shift can be found due to the low content of $\mathrm{Co}$. As a comparison, the diffraction pattern of RuCo alloy (Supplementary Fig. 4) is slightly shifted to higher angles compared to those of pristine $\mathrm{Ru}$, corresponding to the lattice contraction resulting from the incorporation of $\mathrm{Co}$ atoms into Ru nanosheets. To better understand the elemental distribution of $\mathrm{Ru}$ and $\mathrm{Co}$ elements, the energy-dispersive $\mathrm{X}$-ray (EDX) spectroscopy mapping and the EDX line scanning profile were collected. As shown in Fig. If, Ru and Co elements were distributed homogeneously throughout the structure. EDX analysis further demonstrates that the NSs are composed of Ru and Co elements with atomic ratios of 94 and 6\% (Supplementary Fig. 5). The atomic ratio of $\mathrm{Ru}$ and $\mathrm{Co}$ was higher than that of the reactants, which was similar to our previous studies ${ }^{29}$.

To precisely investigate the local atomic and electronic structures of the Co-substituted $\mathrm{Ru}$ samples, extended X-ray absorption fine structure (EXAFS) and X-ray absorption nearedge structure (XANES) measurements were conducted. Figure 2a shows the XANES curves at Ru K-edge of Co-substituted Ru NSs. It is found that the $\mathrm{Ru} \mathrm{K}$-edge of the sample slightly shifts to lower energy in comparison to that of $\mathrm{Ru}$ foil reference, suggesting that $\mathrm{Ru}$ in Co-substituted $\mathrm{Ru}$ NSs gets some electrons from Co. From the XANES spectra of Co K-edge in Fig. 2b, we found that the absorption threshold of Co-substituted Ru NSs is between those of Co foil and Co-O, indicating that $\mathrm{Co}$ in $\mathrm{Co}-$ substituted $\mathrm{Ru}$ NSs is positive charged. In order to further investigate the interaction between $\mathrm{Ru}$ and $\mathrm{Co}$, Fourier transform
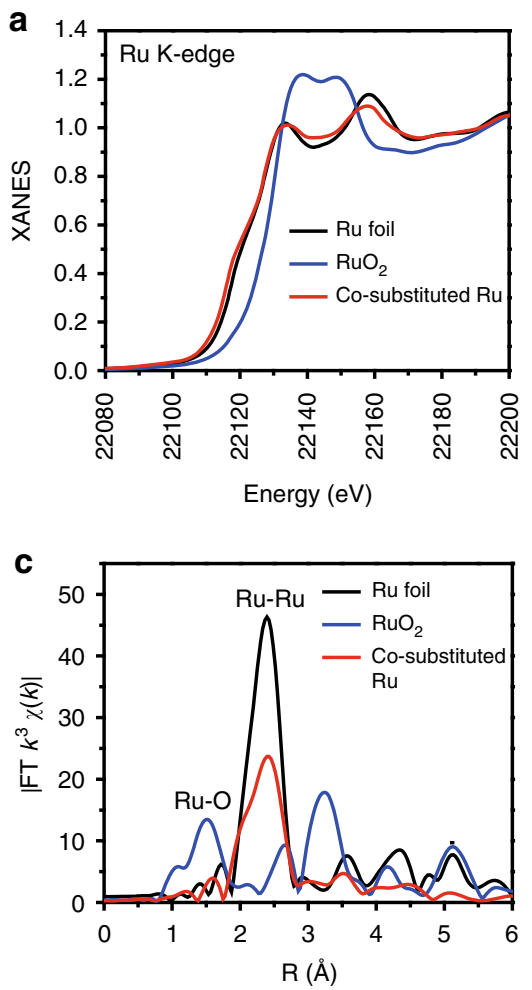

(FT) EXAFS analysis is performed at Ru K-edge (Fig. 2c) and Co K-edge (Fig. 2d), respectively. In FT-EXAFS spectra of Ru K-edge, there is no peak related to $\mathrm{Ru}-\mathrm{O}$ scattering, and the peak at $2.39 \AA$ (slightly shorter than $2.41 \AA$ of $\mathrm{Ru}-\mathrm{Ru}$ in $\mathrm{Ru}$ foil) is due to $\mathrm{Ru}-\mathrm{Ru} / \mathrm{Co}$ contribution. In FT-EXAFS curves of Co K-edge (Fig. 2d), Co-O coordination is not observed and the peak at 2.39 $\AA$ results from $\mathrm{Co}-\mathrm{Ru}$ interaction. It is necessary to mention that the position of $\mathrm{Co}-\mathrm{Ru}$ peaks is obviously positive than that of Co-Co in Co foil $(2.16 \AA)$. This result indicates the absence of Co-Co scattering in Co-substituted Ru NSs and demonstrates the monodispersing of Co atoms in Co-substituted Ru NSs.

Catalytic properties. To demonstrate the superior catalytic properties, the electrocatalytic activities of the as-obtained Cosubstituted Ru NSs toward HER were investigated in hydrogensaturated $1 \mathrm{M} \mathrm{KOH}$ solution using a three-electrode system (see Supporting Information for details, Supplementary Fig. 6-8). As a comparison, commercial $\mathrm{Pt} / \mathrm{C}, \mathrm{Ru} / \mathrm{C}, \mathrm{RuCo}$ and $\mathrm{RuCo}_{2}$ alloy (Supplementary Fig. 9, 10) were measured under the same conditions. Figure $3 a$ shows the linear sweep voltammetry (LSV) curves for the HER over the Co-substituted $\mathrm{Ru}, \mathrm{RuCo}$ alloy, $\mathrm{Ru} / \mathrm{C}$ and $\mathrm{Pt} / \mathrm{C}$ catalysts. As can be seen from Fig. 3a and Supplementary Fig. 10a, the Co-substituted $\mathrm{Ru}$ catalyst shows the highest catalytic activity than that of $\mathrm{RuCo}_{2}, \mathrm{RuCo}, \mathrm{Ru} / \mathrm{C}$ and $\mathrm{Pt} /$ $\mathrm{C}$ catalysts. This result indicates that the monatomic substitution of Co can greatly enhance the catalytic activity of HER, while the presence of $\mathrm{Co}-\mathrm{Co}$ bond in $\mathrm{RuCo}$ and $\mathrm{RuCo}_{2}$ alloy would lead to the decrease of the catalytic activity. Strikingly, the Co-substituted $\mathrm{Ru}$ NSs only required an overpotential as low as $13.0 \mathrm{mV}$ to achieve current density of $10 \mathrm{~mA} \mathrm{~cm}^{-2}$, which is much lower than that of $\mathrm{RuCo}_{2}(144 \mathrm{mV}), \mathrm{RuCo}(40.0 \mathrm{mV}), \mathrm{Ru} / \mathrm{C}(92.5 \mathrm{mV})$ and $\mathrm{Pt} / \mathrm{C}(56.5 \mathrm{mV})$ catalysts (Fig. $3 \mathrm{~b})$ and many other Pt-free catalysts (Supplementary Table 1), suggesting its superior HER activity. The HER kinetics of the above catalysts were also calculated via corresponding Tafel plots. As shown in Fig. $3 c$ and
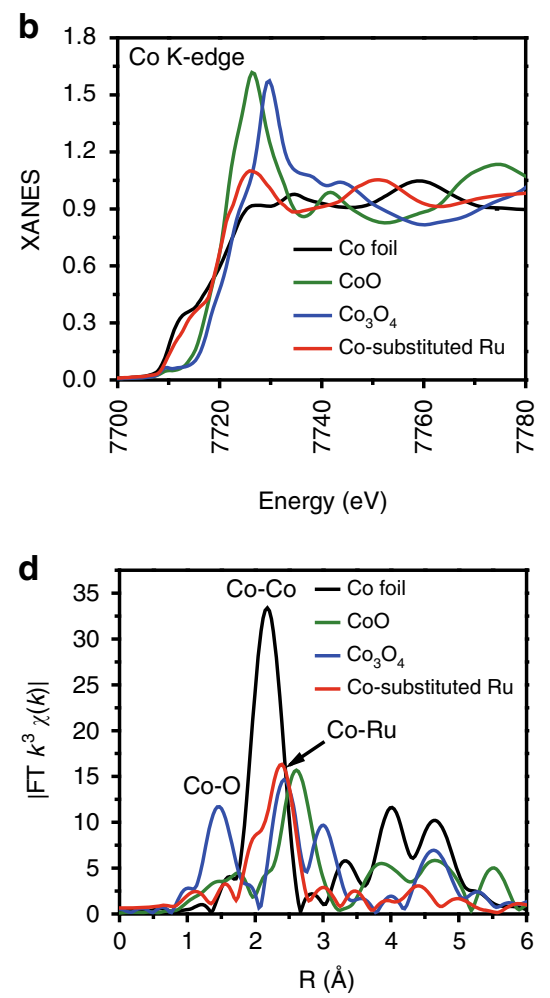

Fig. 2 Structure determination of Co-substituted Ru by XAFS. XANES spectra of Co-substituted Ru for a Ru K-edge and b Co K-edge. Fourier transformed (FT) $k^{3}$-weighted $\chi(k)$-function of the EXAFS spectra for c Ru K-edge and $\mathbf{d}$ Co K-edge 
a

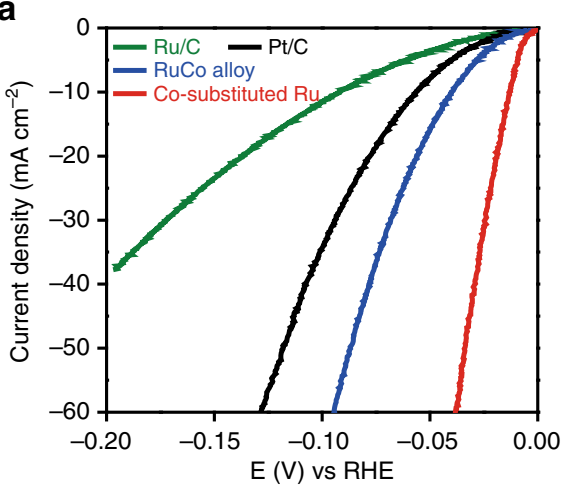

b

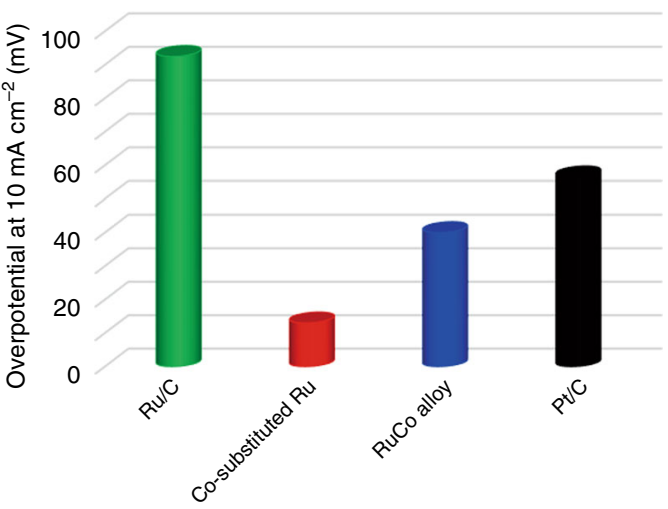

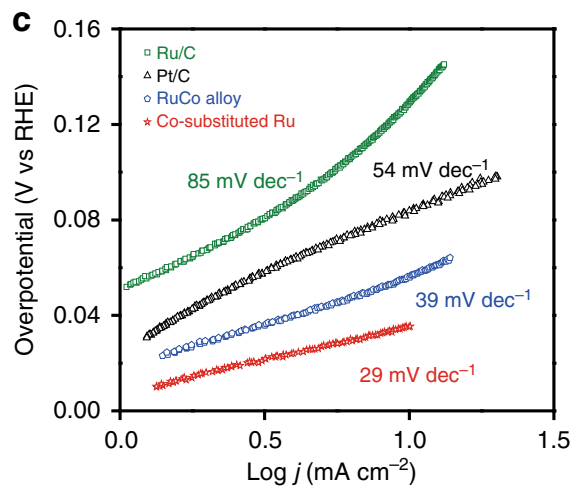
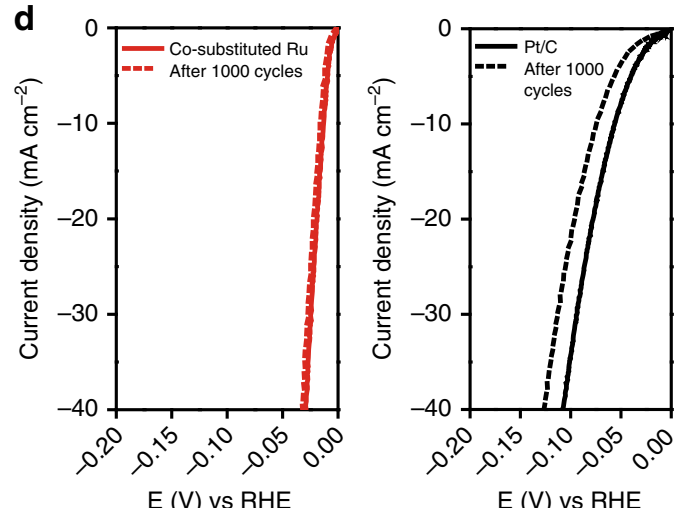

E (V) vs RHE

Fig. 3 Comparison of catalytic ability of Ru/C, Pt/C, RuCo alloy and Co-substituted Ru. a LSV curves, scan rate: $5 \mathrm{mV} \mathrm{s}^{-1}$. b Overpotentials at $10 \mathrm{~mA} \mathrm{~cm}^{-2}$ of Ru/C, Pt/C, RuCo and Co-substituted Ru. c Tafel plots of Ru/C, Pt/C, RuCo and Co-substituted Ru in $1 \mathrm{M} \mathrm{KOH}$. d Durability test of Pt/C and Co-substituted $\mathrm{Ru}$, the LSV curves measured before and after 1000 cycles at a scan rate of $5 \mathrm{mV} \mathrm{s}^{-1}$

Supplementary Fig. 10b, the Tafel slope of the Co-substituted Ru is $29 \mathrm{mV} \mathrm{dec}-1$, which is much smaller than that of $\mathrm{Ru} / \mathrm{C}(85 \mathrm{mV}$ $\left.\mathrm{dec}^{-1}\right), \mathrm{Pt} / \mathrm{C}\left(54 \mathrm{mV} \mathrm{dec}{ }^{-1}\right), \mathrm{RuCo}_{2}\left(122 \mathrm{mV} \mathrm{dec}^{-1}\right)$ and $\mathrm{RuCo}$ alloy $\left(39 \mathrm{mV} \mathrm{dec}^{-1}\right)$, demonstrating its more efficient kinetics of hydrogen evolution. We estimated the turnover frequency (TOF) of the above catalysts to further investigate their intrinsic HER activities (Supplementary Fig. 11, 12). The TOF values of Cosubstituted $\mathrm{Ru}$ at 30 and $60 \mathrm{mV}$ are $2.15 \mathrm{H}_{2} \mathrm{~s}^{-1}$ and $6.39 \mathrm{H}_{2} \mathrm{~s}^{-1}$, respectively, both of which are much higher than those of $\mathrm{Pt} / \mathrm{C}$ $\left(0.43 \mathrm{H}_{2} \mathrm{~s}^{-1}\right.$ at $30 \mathrm{mV} ; 1.69 \mathrm{H}_{2} \mathrm{~s}^{-1}$ at $\left.60 \mathrm{mV}\right), \mathrm{Ru} / \mathrm{C}\left(0.41 \mathrm{H}_{2} \mathrm{~s}^{-1}\right.$ at $30 \mathrm{mV} ; 1.23 \mathrm{H}_{2} \mathrm{~s}^{-1}$ at $\left.60 \mathrm{mV}\right)$ and RuCo alloy $\left(0.51 \mathrm{H}_{2} \mathrm{~s}^{-1}\right.$ at $30 \mathrm{mV} ; 2.07 \mathrm{H}_{2} \mathrm{~s}^{-1}$ at $60 \mathrm{mV}$ ), further revealing that Cosubstituted Ru NSs have a superior intrinsic HER activity.

To evaluate the durability of the Co-substituted $\mathrm{Ru}$ NSs, longterm cyclic voltammetry between $0 \mathrm{~V}$ and $-0.20 \mathrm{~V}$ (vs RHE) was examined. The commercial $\mathrm{Pt} / \mathrm{C}$ catalyst was used as a reference under the same conditions. As shown in Fig. 3d, the polarization curve of $\mathrm{Pt} / \mathrm{C}$, negatively shifted about $47 \mathrm{mV}$ after 1000 cycles at a current density of $10 \mathrm{~mA} \mathrm{~cm}^{-2}$, indicating an obvious decrease in the electrocatalytic activity. As for Co-substituted $\mathrm{Ru}$ NSs, the polarization curve of Co-substituted $\mathrm{Ru}$ after 1000 continuous cycles was almost overlapped with the initial one, suggesting the durability of Co-substituted $\mathrm{Ru}$ NSs under the electrocatalytic processes. The durability of the Co-substituted Ru NSs was further evaluated by chronopotentiometry at a constant current density of $10 \mathrm{~mA} \mathrm{~cm}^{-2}$ in $1.0 \mathrm{M} \mathrm{KOH}$ electrolytes. As shown in Supplementary Fig. 13, the Co-substituted $\mathrm{Ru}$ catalyst shows negligible degradation in the course of $72,000 \mathrm{~s}$. The TEM image (Supplementary Fig. 14) and EDX results (Supplementary Fig. 15) show no obvious morphology and composition change for Co-substituted $\mathrm{Ru}$ catalyst even after 1000 cycle durability test. Furthermore, we utilize the in situ XAFS measurements (Fig. 4a) to monitor the atomic structure evolution of $\mathrm{Ru}$ and $\mathrm{Co}$ in Co-substituted $\mathrm{Ru}$ catalyst during the catalytic reaction process. As shown in Fig. 4b-e, no obvious change can be found from the in situ XANES spectra and EXAFS spectra for the Ru K-edge and Co K-edge at different voltages, demonstrating the high stability of the local atomic structure of Co-substituted $\mathrm{Ru}$ catalyst. Therefore, introducing monatomic Co into $\mathrm{Ru}$ lattice can greatly boost the electrocatalytic activity and durability toward HER, making it much more active than $\mathrm{Pt} / \mathrm{C}$ catalyst and even all the Pt-free catalysts.

DFT studies on Co-substituted Ru catalysts. We further performed DFT calculations to gain insights into the atomic Co substitution effect on the HER activity. Generally, the typical process of HER $\left(2 \mathrm{H}^{+}+2 \mathrm{e}^{-} \rightarrow \mathrm{H}_{2}\right)$ in alkaline condition is a twostep electrochemical process taking place on the catalytic active center that finally generates gaseous hydrogen ${ }^{30}$. First is the electrochemical hydrogen adsorption (Volmer step; Eq. (1)

$$
\mathrm{H}_{2} \mathrm{O}+\mathrm{M}+\mathrm{e}^{-} \rightarrow \mathrm{M}-\mathrm{H}^{*}+\mathrm{OH}^{-},
$$

followed by the electrochemical desorption (Heyrovsky step; Eq. (2)) or chemical desorption (Tafel step; Eq. (3))

$$
\begin{gathered}
\mathrm{M}-\mathrm{H}^{*}+\mathrm{H}_{2} \mathrm{O}+\mathrm{e}^{-} \rightarrow \mathrm{M}+\mathrm{H}_{2}+\mathrm{OH}^{-}, \\
2 \mathrm{M}-\mathrm{H}^{*} \rightarrow 2 \mathrm{M}+\mathrm{H}_{2} .
\end{gathered}
$$

Obviously, the chemical adsorption and desorption of $\mathrm{H}$ atoms on the catalyst surface should be neither too strong nor too weak as they are competitive processes. According to the Sabatier ${ }^{31}$ principle, in the viewpoint of thermodynamics, a good HER 
a

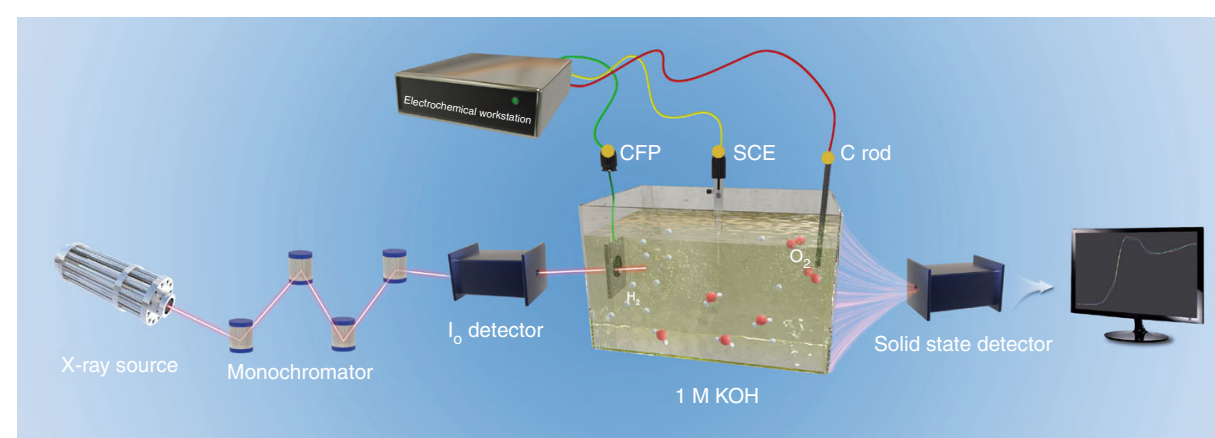

b

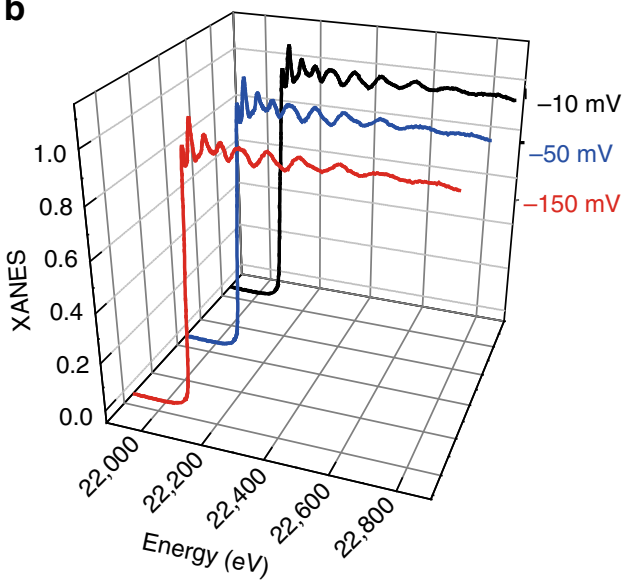

d

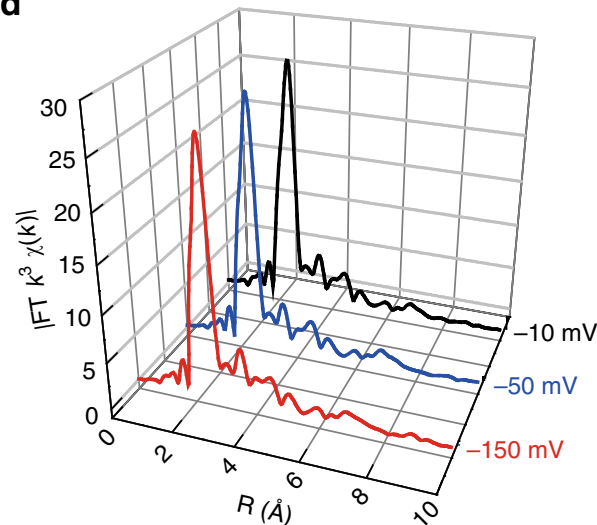

C
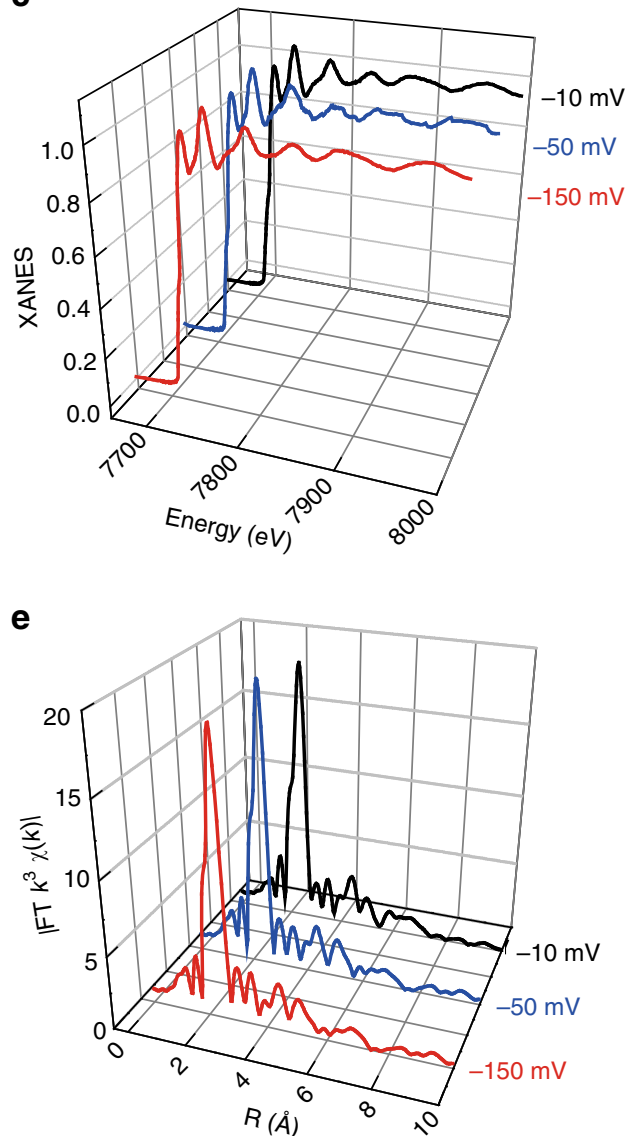

Fig. 4 In situ XAFS measurements for Co-substituted Ru catalyst. a Schematic illustration of in situ XAS measurements for Co-substituted Ru. CFP and SCE represent carbon fiber paper and saturated calomel electrode, respectively. XANES spectra of Co-substituted Ru for the $\mathbf{b}$ Ru K-edge and $\mathbf{c}$ Co K-edge at different voltages. EXAFS spectra for the $\mathbf{d}$ Ru K-edge and e Co K-edge at different voltages

catalyst should possess a hydrogen adsorption-free energy $\left(\Delta G_{\mathrm{H}^{*}}\right.$ as an activity descriptor) with an optimal value around zero ${ }^{32,33}$. Thus, we first calculated the hydrogen adsorption-free energy on $\mathrm{Ru}$ (0001) surfaces with or without Co substitution. The results showed that the $\Delta G_{\mathrm{H}^{*}}$ values after Co atom substitution are more negative than that of pristine $\mathrm{Ru}$ (Fig. 5a), indicating stronger hydrogen binding abilities after Co atom substitution, which should lead to the obstacle of thermodynamic $\mathrm{H}^{*}$ desorption process in the Heyrovsky step. Actually, relatively inert $\mathrm{H}_{2} \mathrm{O}$ molecules are the reacting species for HER in alkaline media, and hence the water dissociation kinetic of Volmer step, namely the energy barrier $\left(\Delta G_{\mathrm{w}}\right)$ of $\mathrm{O}-\mathrm{H}$ bond cleavage, is usually more crucial to the overall reaction rate ${ }^{34}$. As shown in Fig. $5 \mathrm{~b}$, the favorable hydrogen desorption process is the Tafel step with $\Delta G_{\mathrm{w}}$ value of $13.42 \mathrm{kcal} \mathrm{mol}^{-1}$, being much lower than that of Heyrovsky step $\left(\Delta G_{\mathrm{w}}=22.47 \mathrm{kcal} \mathrm{mol}^{-1}\right)$. Moreover, it is also obviously lower than that of the Volmer step $\left(\Delta G_{\mathrm{w}}=19.28 \mathrm{kcal}\right.$ $\mathrm{mol}^{-1}$ ), indicating that the water dissociation is indeed the ratelimiting process of HER in this material. Expectedly, the energy barrier of water dissociation has remarkably reduced after single Co atom substitution (denoted as RuCo1) compared with that of pristine $\mathrm{Ru}\left(\Delta G_{\mathrm{w}}=26.48 \mathrm{kcal} \mathrm{mol}^{-1}\right)$, being consistent with the experimental observation. However, when increasing the substituted Co atoms to two (denoted as RuCo2) and three (denoted as $\mathrm{RuCo} 3$ ) per unit cell, the energy barriers instead increase to 23.01 and $21.89 \mathrm{kcal} \mathrm{mol}^{-1}$ (Fig. 5a, c), respectively, meaning that more Co substitution can lead to sluggish water dissociation due to the destruction of single atom dispersion. Moreover, the $\mathrm{OH}$ binding energy $\left(\Delta E_{\mathrm{OH}}\right)$ gradually increases with the addition of Co atoms (Fig. 5a), which should result from the increasing electron density in the saddle points (groove formed by the three nearest atoms) around Co atom (Fig. 5d). This change, on one 
a

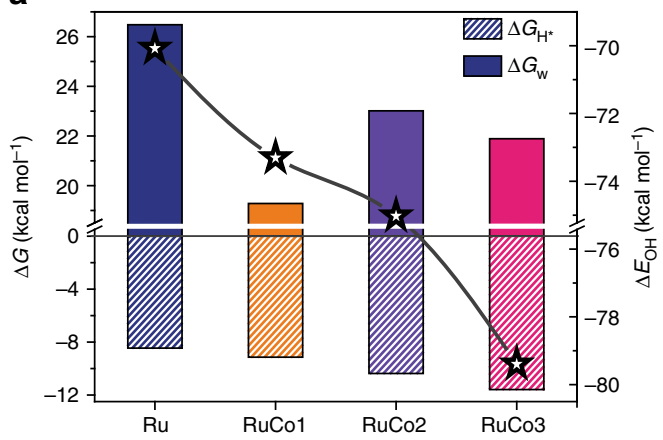

C

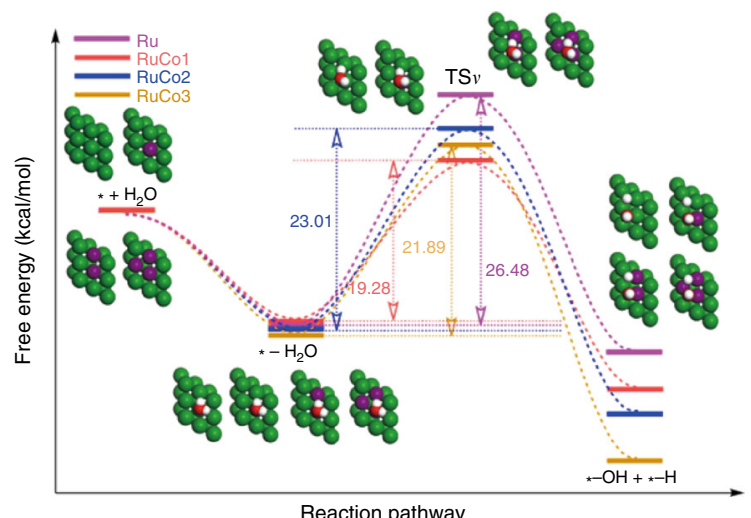

b

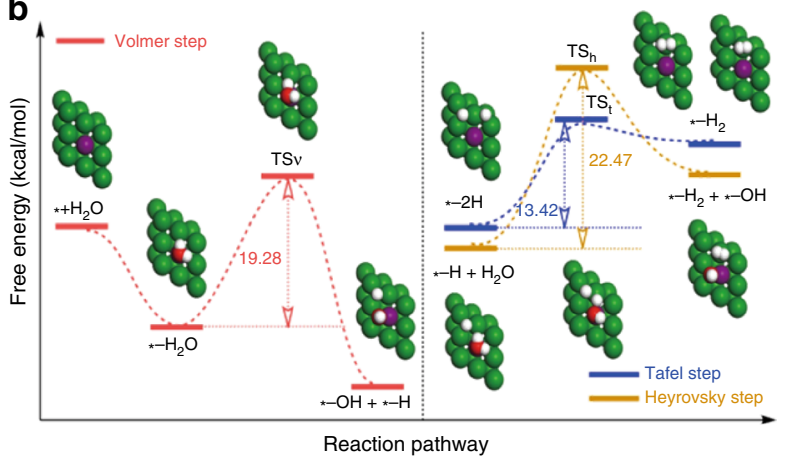

d

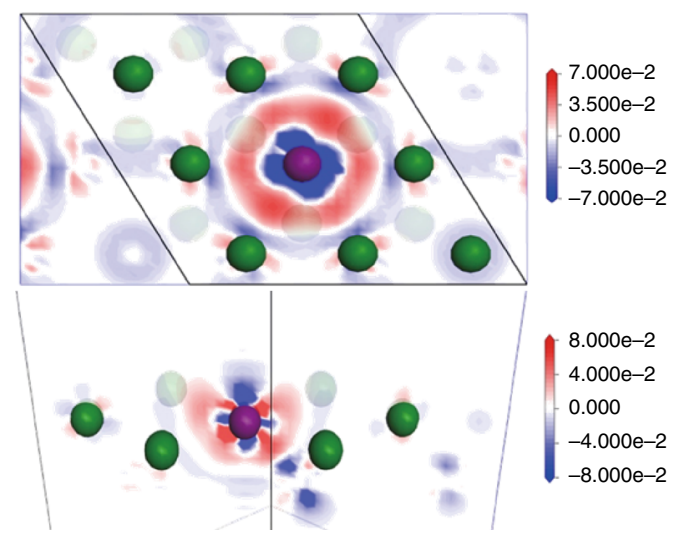

Fig. 5 DFT calculations to gain insights into the atomic Co substitution effect on the HER activity. a The hydrogen adsorption-free energy $\left(\Delta G_{H}{ }^{\star}\right.$ ), water dissociation energy barrier $\left(\Delta G_{\mathrm{w}}\right)$ and $\mathrm{OH}$ binding energy $\left(\Delta E_{\mathrm{OH}}\right)$ on various metal surfaces with different amount of Co substitution. $\mathbf{b}$ Free energy diagrams of the three elementary processes of HER on RuCo1 including atomic configurations of reactant initial states, intermediate state, final states and additional transition states (TSv, TSh and TSt represent the transition states of Volmer, Heyrovsky and Tafel step, respectively). c Free energy diagrams of the Volmer steps of HER on various metal surfaces with different amounts of Co substitution including atomic configurations of reactant initial states, intermediate state, final states and additional transition states (TSv). d Top view (up) and side view (down) of the electron density change plots after single Co atom substitution. Color: green, Ru; purple, $\mathrm{Co}$; red, $\mathrm{O}$; white, $\mathrm{H}$

hand, benefits for boosting the cleavage of $\mathrm{O}-\mathrm{H}$ bond, but on the other hand, causes adverse $\mathrm{OH}$ to be desorbed and then blocks the consequent reactions. Therefore, a moderate $\Delta E_{\mathrm{OH}}$ value of the single atom doped structure can keep a favorable balance between facilitating water dissociation and preventing the active sites from inhibiting by the high-affinity hydroxides and finally accelerates reaction kinetics.

\section{Discussion}

In summary, Co-substituted $\mathrm{Ru}$ NSs as highly efficient Pt-free HER catalysts were successfully synthesized. The structure of Co-substituted Ru NSs was characterized by aberration-corrected HAADF-STEM and XAFS measurements, indicating the atomically dispersed $\mathrm{Co}$ into $\mathrm{Ru}$ lattice. More importantly, the Cosubstituted Ru NSs exhibit superior catalytic performance for HER in alkaline, which is much better than the activity of commercial Pt/C catalyst and other Pt-free catalysts. Theoretical calculations suggested that single Co atom substitution significantly reduce energy barrier of water dissociation, leading to the outstanding HER performance. Our findings not only present new opportunities to design highly active HER catalysts at atomic scale, but also advance our understanding of HER through theoretical insights.

\section{Methods}

Characterization. The powder XRD patterns were recorded with a Bruker $\mathrm{D} 8$-advance X-ray powder diffractometer with monochromatized CuKa radiation $(\lambda=1.5406 \AA)$. The morphology and structure of the obtained products were determined using a Hitachi model H-800 TEM and a FEI Tecnai G2 F20 S-Twin high-resolution TEM operated at $200 \mathrm{kV}$. The HAADF-STEM images were performed using a Titan 80-300 scanning/transmission electron microscope operated at $300 \mathrm{kV}$, equipped with a probe spherical aberration corrector.

Preparation of Co-substituted Ru and RuCo alloy. In a typical synthesis of Co-substituted $\mathrm{Ru}$ nanosheets, $40 \mathrm{mg}$ of $\mathrm{Ru}_{3}(\mathrm{CO})_{12}, 5.2 \mathrm{mg}$ of $\mathrm{Co}(\mathrm{acac})_{2}, 500 \mathrm{mg}$ of glucose and $100 \mathrm{mg}$ of citric acid monohydrate were dissolved in $20 \mathrm{~mL}$ of oleylamine and $10 \mathrm{~mL}$ of 1-heptanol mixed solvents, followed by vigorous stirring for $2 \mathrm{~h}$. Then, the resulting homogeneous solution was transferred into a $50 \mathrm{~mL}$ Teflon-lined autoclave and heated at $180^{\circ} \mathrm{C}$ for $12 \mathrm{~h}$. After cooling down to the room temperature, the black precipitate was obtained by centrifugation, and washed with the ethanol-cyclohexane mixture for several times. Finally, the Co-substituted $\mathrm{Ru}$ nanosheets were obtained. The preparation of RuCo alloy was the same as Co-substituted $\mathrm{Ru}$ nanosheets except that the amount of Co was changed to $48 \mathrm{mg}$.

XAFS measurements and analysis. The XAFS (Ru K-edge and Co K-edge) spectra were collected at BL14W1 station in Shanghai Synchrotron Radiation Facility (SSRF, the storage rings were operated at $3.5 \mathrm{GeV}$ with a maximum current of $250 \mathrm{~mA}$ ). The data were collected at room temperature in transmission mode (Si(311) monochromator for Ru K-edge and $\mathrm{Si}(111)$ monochromator for $\mathrm{Co}$ $\mathrm{K}$-edge). All samples were pelletized as disks of $13 \mathrm{~mm}$ diameter using graphite powder as a binder (ground thoroughly by mortar and pestle). The acquired EXAFS data were processed according to the standard procedures using the ATHENA module implemented in the IFEFFIT software packages. The EXAFS spectra were obtained by subtracting the post-edge background from the overall absorption and then normalizing with respect to the edge-jump step. Subsequently, $\chi(k)$ data in the $k$-space were Fourier transformed to real (R) space using a hanning windows $\left(\mathrm{dk}=1.0 \AA^{-1}\right)$ to separate the EXAFS contributions from different coordination shells. 
Electrochemical measurements. The electrochemical measurements were conducted on a three-electrode system (Supplementary Fig. 6) controlled by a potentiostat (V3, Princeton Applied Research). The catalyst-modified glassy carbon (GC) electrodes, graphite rod and saturated calomel electrode (SCE) were used as working electrode (geometric area of a working electrode: $0.196 \mathrm{~cm}^{2}$ ), counter electrode (CE) and reference electrode (the SCE was calibrated, Supplementary Fig. 7), respectively. Before test, GC substrate electrodes were polished with 0.3 and $0.05 \mathrm{~mm} \mathrm{Al} \mathrm{O}_{3}$ slurry and then sonicated in ethanol and water each for several times to make it clean. For preparation of the electrode materials, $2 \mathrm{mg}$ of catalyst powder was dispersed in isopropanol/distilled water (Milli-Q, volume ratio, 1:1) mixed solution containing $0.05 \mathrm{wt} \%$ of Nafion. The mixture was ultrasonicated for about $2 \mathrm{~h}$ to generate a homogeneous ink. After that, $15 \mu \mathrm{L}$ of ink solutions was transferred onto the GC rotating disk electrode and then dried at room temperature. We optimize the catalytic activity of the Co-substituted Ru catalysts by tuning the loading mass before electrochemical test. As shown in Supplementary Fig. 8, the optimal loading of the catalysts was $0.153 \mathrm{mg} \mathrm{cm}^{-2}$ in $1.0 \mathrm{M} \mathrm{KOH}$. The hydrogen-saturated $1 \mathrm{M} \mathrm{KOH}$ aqueous solutions were used as the electrolytes before each test. The scan rate was set to $5 \mathrm{mV} \mathrm{s}^{-1}$ for LSV measurements. All polarization curves were corrected for the iR compensation (the specific percentage of the correction is $100 \%$ ). The electrochemical tests were performed at room temperature.

The TOF values can be calculated by the following equation: TOF $=I /(2 F n)$, where $I$ represents the current, $F$ represents the Faraday constant and $n$ represents the number of active sites. Forming $\mathrm{H}_{2}$ needs two electrons that leads to the factor of $1 / 2$ in this equation. Here, the number of active sites can be qualified by the following equation ${ }^{19,35-37:} n=Q_{\mathrm{Cu}} / 2 F$, where $Q_{\mathrm{Cu}}$ represents the underpotential deposition (UPD, Supplementary Fig. 11) copper stripping charge $\left(\mathrm{Cu}\right.$ upd $\left.\rightarrow \mathrm{Cu}^{2+}+2 \mathrm{e}^{-}\right)$.

Details of DFT calculations. The spin polarization DFT calculations were performed by the $\mathrm{Dmol}^{3}$ module in Materials Studio 5.5 package and generalized gradient approximation with Perdew-Becke-Ernzerhof (PBE) was used for the exchange-correlation functional. The double numerical plus polarization (DNP) basis set were adopted, while an accurate DFT semi-core pseudopots (DSPP) was employed for the metal atoms. All of the models are calculated in periodically boxes with a vacuum slab of $15 \AA$ to separate the interaction between periodic images. The simulated unit cell is hexagonal with $8.12 \times 8.12 \times 19.28 \AA^{3}$. The transition state search was performing with a linear synchronous transit (LST), followed by repeated conjugate gradient minimizations and quadratic synchronous transit (QST) maximizations until a transition state has been located. All the transition state configurations were confirmed through the frequency analysis. The energy, gradient and displacement convergence criteria were set as $1 \times 10^{-5} \mathrm{Ha}$, $2 \times 10^{-3} \AA$ and $5 \times 10^{-3} \AA$, respectively. The Gibbs free energy of each elementary step was calculated as

$$
\Delta G=\Delta E+\Delta \mathrm{ZPE}-T \Delta S,
$$

where $\Delta E$ is the reaction energy calculated using the spin polarization DFT method. $\triangle \mathrm{ZPE}$ and $\Delta S$ are the changes in zero-point energies and entropy during the reaction, respectively. Particularly, as the vibrational entropy of $\mathrm{H}^{*}$ in the adsorbed state is small, the entropy of adsorption of $1 / 2 \mathrm{H}_{2}$ is $\Delta S_{\mathrm{H}} \approx-0.5 S_{0 \mathrm{H} 2}$, where $S_{0 \mathrm{H} 2}$ is the entropy of $\mathrm{H}_{2}$ in the gas phase at the standard conditions.

\section{Data availability}

The data supporting this study are available from the authors on reasonable request.

Received: 13 June 2018 Accepted: 24 October 2018

Published online: 23 November 2018

\section{References}

1. Yin, H. et al. Ultrathin platinum nanowires grown on single-layered nickel hydroxide with high hydrogen evolution activity. Nat. Commun. 6, 6430-6437 (2015).

2. Wang, P. et al. Phase and interface engineering of platinum-nickel nanowires for efficient electrochemical hydrogen evolution. Angew. Chem. Int. Ed. 55, 12859-12863 (2016).

3. Duan, H. et al. High-performance $\mathrm{Rh}_{2} \mathrm{P}$ electrocatalyst for efficient water splitting. J. Am. Chem. Soc. 139, 5494-5502 (2017).

4. Gong, M. et al. A mini review on nickel-based electrocatalysts for alkaline hydrogen evolution reaction. Nano Res. 9, 28-46 (2016).

5. Lu, S. \& Zhuang, Z. Electrocatalysts for hydrogen oxidation and evolution reactions. Sci. China Mater. 59, 217-238 (2016).

6. Yu, XY., Yu, L., Wu, HB. \& Lou, XW. Formation of nickel sulfide nanoframes from metal-organic frameworks with enhanced pseudocapacitive and electrocatalytic properties. Angew. Chem. Int. Ed. 127, 5421-5425 (2015).
7. $\mathrm{Xu}$, J. et al. Ultrasmall $\mathrm{Cu}_{7} \mathrm{~S}_{4} @ \mathrm{MoS}_{2}$ hetero-nanoframes with abundant active edge sites for ultrahigh-performance hydrogen evolution. Angew. Chem. Int. Ed. 55, 6502-6505 (2016).

8. Xie, J. et al. Defect-rich $\mathrm{MoS}_{2}$ ultrathin nanosheets with additional active edge sites for enhanced electrocatalytic hydrogen evolution. Adv. Mater. 25, 5807-5813 (2013).

9. Yang, L. et al. Hierarchical spheres constructed by defect-rich $\mathrm{MoS}_{2} /$ carbon nanosheets for efficient electrocatalytic hydrogen evolution. Nano Energy 22, 490-498 (2016).

10. Zhang, L., Liu, B., Zhang, N. \& Ma, M. Electrosynthesis of $\mathrm{Co}_{3} \mathrm{O}_{4}$ and Co $(\mathrm{OH})_{2}$ ultrathin nanosheet arrays for efficient electrocatalytic water splitting in alkaline and neutral media. Nano Res. 11, 323-333 (2018).

11. Tian, J., Liu, Q., Asiri, A. M. \& Sun, X. Self-supported nanoporous cobalt phosphide nanowire arrays: an efficient 3D hydrogen-evolving cathode over the wide range of $\mathrm{pH}$ 0-14. J. Am. Chem. Soc. 136, 7587-7590 (2014).

12. Gao, M. et al. An efficient molybdenum disulfide/cobalt diselenide hybrid catalyst for electrochemical hydrogen generation. Nat. Commun. 6, 5982-5988 (2015).

13. Deng, J., Ren, P., Deng, D. \& Bao, X. Enhanced electron penetration through an ultrathin graphene layer for highly efficient catalysis of the hydrogen evolution reaction. Angew. Chem. Int. Ed. 54, 2100-2104 (2015).

14. Duan, J. et al. 3D WS $\mathrm{W}_{2}$ nanolayers@ heteroatom-doped graphene films as hydrogen evolution catalyst electrodes. Adv. Mater. 27, 4234-4241 (2015).

15. Yang, J. et al. Porous molybdenum phosphide nano-octahedrons derived from confined phosphorization in UIO-66 for efficient hydrogen evolution. Angew. Chem. Int. Ed. 128, 13046-13050 (2016).

16. Ledendecker, M. et al. Stability and activity of non-noble-metal-based catalysts toward the hydrogen evolution reaction. Angew. Chem. Int. Ed. 129, 9899-9903 (2017)

17. Xu, X., Nosheen, F. \& Wang, X. Ni-decorated molybdenum carbide hollow structure derived from carbon-coated metal-organic framework for electrocatalytic hydrogen evolution reaction. Chem. Mater. 28, 6313-6320 (2016).

18. Tang, Y. et al. Porous molybdenum-based hybrid catalysts for highly efficient hydrogen evolution. Angew. Chem. Int. Ed. 54, 12928-12932 (2015).

19. Mahmood, J. et al. An efficient and $\mathrm{pH}$-universal ruthenium-based catalyst for the hydrogen evolution reaction. Nat. Nanotechnol. 12, 441-446 (2017).

20. Kibsgaard, J. \& Jaramillo, T. Molybdenum phosphosulfide: an active, acidstable, earth-abundant catalyst for the hydrogen evolution reaction. Angew. Chem. Int. Ed. 53, 14433-14437 (2014).

21. Popczun, E. et al. Highly active electrocatalysis of the hydrogen evolution reaction by cobalt phosphide nanoparticles. Angew. Chem. Int. Ed. 53, 5427-5430 (2014).

22. Popczun, E. et al. Nanostructured nickel phosphide as an electrocatalyst for the hydrogen evolution reaction. J. Am. Chem. Soc. 135, 9267-9270 (2013).

23. Chen, W. et al. Rational design of single molybdenum atoms anchored on $\mathrm{N}$ doped carbon for effective hydrogen evolution reaction. Angew. Chem. Int. Ed. 129, 16302-16306 (2017).

24. Fei, H. et al. Atomic cobalt on nitrogen-doped graphene for hydrogen generation. Nat. Commun. 6, 8668-8676 (2015).

25. Qiu, H. et al. Nanoporous graphene with single-atom nickel dopants: an efficient and stable catalyst for electrochemical hydrogen production. Angew. Chem. Int. Ed. 54, 14031-14035 (2015)

26. $\mathrm{Xu}$, J. et al. Boosting the hydrogen evolution performance of ruthenium clusters through synergistic coupling with cobalt phosphide. Energy Environ. Sci. 11, 1819-1827 (2018)

27. Li, H. et al. Amorphous nickel-cobalt complexes hybridized with 1T-phase molybdenum disulfide via hydrazine induced phase transformation for water splitting. Nat. Commun. 8, 15377-15387 (2017).

28. Su, J. et al. Ruthenium-cobalt nanoalloys encapsulated in nitrogen-doped graphene as active electrocatalysts for producing hydrogen in alkaline media. Nat. Commun. 8, 14969-14978 (2017).

29. Mao, J. et al. Rational control of the selectivity of a ruthenium catalyst for hydrogenation of 4-nitrostyrene by strain regulation. Angew. Chem. Int. Ed. 56, 11971-11975 (2017)

30. Zheng, Y., Jiao, Y., Jaroniec, M. \& Qiao, S. Advancing the electrochemistry of the hydrogen-evolution reaction through combining experiment and theory. Angew. Chem. Int. Ed. 54, 52-65 (2015).

31. Sabatier, P. La catalyse en chimie organique (Librairie Polytechnique, Paris et Liège, Paris, 1920).

32. Deng, J. et al. Multiscale structural and electronic control of molybdenum disulfide foam for highly efficient hydrogen production. Nat. Commun. 8, 14430-14437 (2017).

33. Sku'lason, E. et al. Modeling the electrochemical hydrogen oxidation and evolution reactions on the basis of density functional theory calculations. J. Phys. Chem. C 114, 18182-18197 (2010). 
34. Zheng, Y. et al. High electrocatalytic hydrogen evolution activity of an anomalous ruthenium catalyst. J. Am. Chem. Soc. 138, 16174-16181 (2016)

35. Trasatti, S. \& Petrii, O. Real surface area measurements in electrochemistry. Pure Appl. Chem. 63, 711-734 (1991).

36. Ohyama, J. et al. Size specifically high activity of Ru nanoparticles for hydrogen oxidation reaction in alkaline electrolyte. J. Am. Chem. Soc. 135, 8016-8021 (2013).

37. Green, C. \& Kucernak, A. Determination of the platinum and ruthenium surface areas in platinum-ruthenium alloy electrocatalysts by underpotential deposition of copper. I. Unsupported catalysts. J. Phys. Chem. B 106, 1036-1047 (2002).

\section{Acknowledgements}

This work was supported by the China Ministry of Science and Technology under Contract of 2016YFA (0202801), the National Natural Science Foundation of China (21521091, 21390393, U1463202, 21471089, 21671117) and the Beijing Natural Science Foundation (2174081). We thank the BL14W1 in SSRF, and BL10B and BL12B in NSRL for XAS measurement.

\section{Author contributions}

D.W., Y.L. and J.M. conceived the idea for the project. J.M., J.P. and Y.H. conducted material synthesis. D.H. and J.P. performed structural characterizations and catalytic test. W.C., J.P. and Z.Z. conducted XAFS measurement and analyzed data. C.H. performed DFT calculations. J.M., J.P., D.W., C.C. and Q.P. discussed the catalytic results. D.W., J.M., C.H., W.C. and Y.L. drafted the manuscript. J.M., C.H. and J.P. contributed equally to this work. All authors discussed and commented on the manuscript.

\section{Additional information}

Supplementary Information accompanies this paper at https://doi.org/10.1038/s41467018-07288-6.

Competing interests: The authors declare no competing interests.

Reprints and permission information is available online at http://npg.nature.com/ reprintsandpermissions/

Publisher's note: Springer Nature remains neutral with regard to jurisdictional claims in published maps and institutional affiliations.

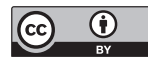

Open Access This article is licensed under a Creative Commons Attribution 4.0 International License, which permits use, sharing, adaptation, distribution and reproduction in any medium or format, as long as you give appropriate credit to the original author(s) and the source, provide a link to the Creative Commons license, and indicate if changes were made. The images or other third party material in this article are included in the article's Creative Commons license, unless indicated otherwise in a credit line to the material. If material is not included in the article's Creative Commons license and your intended use is not permitted by statutory regulation or exceeds the permitted use, you will need to obtain permission directly from the copyright holder. To view a copy of this license, visit http://creativecommons.org/ licenses/by/4.0/.

(C) The Author(s) 2018 